Article

\title{
Instability of Vertical Throughflows in Porous Media under the Action of a Magnetic Field
}

\author{
Florinda Capone * ${ }^{\circledR}$, Roberta De Luca $₫$ and Maurizio Gentile $(1)$ \\ Department of Mathematics and Applications "Renato Caccioppoli", University of Naples Federico II, \\ Via Cinzia, 80126 Naples, Italy; roberta.deluca@unina.it (R.D.L.); m.gentile@unina.it (M.G.) \\ * Correspondence: fcapone@unina.it; Tel.: +39-081-675-645
}

Received: 2 September 2019; Accepted: 29 October 2019; Published: 1 November 2019

\begin{abstract}
The instability of a vertical fluid motion (throughflow) in a binary mixture saturating a horizontal porous layer, uniformly heated from below, uniformly salted from below by one salt and permeated by an imposed uniform magnetic field $\mathbf{H}$, normal to the layer, is analyzed. By employing the order-1 Galerkin weighted residuals method, the critical Rayleigh numbers for the onset of steady or oscillatory instability, have been determined.
\end{abstract}

Keywords: throughflow; fluid motion; steady instability; oscillatory instability; order-1 Galerkin weighted residuals method

\section{Introduction}

The onset of convection in porous media [1] (and the references therein) with particular regard to the porous magneto-hydrodynamic has been, in the past as nowadays, a topic of relevant interest because of the numerous applications in geophysics, astrophysics, industrial processes and since the inhibitory effect of the magnetic field on the onset of convection is used, for instance, in directional solidification of binary alloys. A wide literature can be found on different aspects of porous convection. In particular, in [2] the linear stability of a uniform parallel flow in a horizontal channel with open upper boundary, has been performed; in [3] the linear stability of an unsteady thermal boundary layer in a semi-infinite porous medium is considered; in [4] the thermal instability is analyzed on considering two different boundary conditions on the bounding walls. In [5] the onset of double-diffusive convection with heat generation and absorption effects, has been performed. The more general case when the permeability of the medium is a continuous periodic function of one of the horizontal direction, has been investigated in [6] while in [7] the influence of an embedded, central, solid heat-conducting block on the onset of convection has been analyzed. Reference [8] is a very recent paper dealing with thermal convection in an inclined anisotropic porous layer with internal heat generation. The stabilizing effect of a magnetic field on the onset of convection in an electrically conducting fluid saturating a horizontal porous layer, has been performed in: [9], where the influence of the inertia term in the Darcy equation has been included; [10] on considering the onset of double-diffusive convection; $[11,12]$ in the case of a vertical channel with symmetric and asymmetric wall heating conditions and in a isothermal inclined surface adjacent to a stratified porous medium; $[13,14]$ where the Brinkman law, holding for large pores, has been introduced; [15] where the validity of Linearization Principle guaranteeing the absence of subcritical instability without any restriction on the initial data, has been proved.

Generally, the onset of thermal convection in porous layers may be steady or oscillatory according to the type of the secondary motion arising when the thermal conduction is no longer observable. When the principle of exchange of stability holds, a secondary steady motion (throughflow) arises together with the question of determining until this motion is observable. The problem of analyzing 
the stability/instability of throughflows, has been the subject of many papers. Just to mention a few, in $[16,17]$, the instability of a vertical throughflow has been analyzed on considering, respectively, the effect of viscous dissipation and a non-Newtonian power-law fluid; in [18] the simultaneous influence of a vertical magnetic field and a rotation on the instability of a vertical throughflow in a clear fluid, is considered. In [19-22], the instability of a vertical constant throughflow in a binary mixture is analyzed on considering different effects such as the Brinkman law and a uniform rotation. Horizontally periodic throughflows have been analyzed in [23]. Instability of throughflow in superposed fluids in porous layers has been investigated in [24]. Nonlinear stability, without any restriction on the initial data, has been obtained in [25] for penetrative convection. Non-uniform heating effects on oscillatory instability for a throughflow in a porous medium has been analyzed in [26]. The combined effects of throughflow and magnetic field in micropolar fluids is investigated in [27]. In [28], the double-diffusive instability on a vertical constant throughflow has been examined and the critical Rayleigh number for the onset of instability has been determined by using the order-1 Galerkin method. In [29] the instability a weak bidimensional throughflow on the Darcy-Bénard problem in a finite box, is analyzed. Other fundamental papers concerning the stability/instability of vertical throughflows on considering different effects are [30-36]. The question of determining whenever the secondary motion arising after the loss of stability, is stationary or oscillatory, is a problem of fundamental importance especially in applications involving cloud physics, hydrological/geophysical studies, seabed hydrodynamics, subterranean pollution and many industrial and technological processes. Furthermore, throughflows play an important role in the directional solidification of concentrated alloys, in which mushy zone exists and it is regarded as a porous layer [26].

In the present paper, we want to estimate the simultaneous presence of the stabilizing effects of the magnetic field and of the salt together with the destabilizing effect of the heating from below on the onset of instability for a vertical constant motion. In particular, we investigate the instability of a vertical constant throughflow in a horizontal porous layer, uniformly heated from below and uniformly salted by one chemical from below, embedded in a constant transverse magnetic field. Our aim is to determine the critical Rayleigh thermal number at which instability occurs and to investigate for the kind of secondary motion arising when the (basic) vertical throughflow is no longer observable. Furthermore, we investigate the stabilizing/destabilizing effect of the vertical throughflow on the onset of instability.

The plan of the paper is as follows. Section 2 deals with the introduction of the mathematical problem. Instability analysis via the normal modes is performed in Section 3. The critical Rayleigh thermal number at which steady or oscillatory instability can occur, is determined by employing a 1-order Galerkin weighted residuals method. The paper ends with a Conclusion section containing a table to show some examples in which steady or oscillatory instability sets in and a figure showing the behaviour of $\mathcal{R}_{s}$ (critical thermal Rayleigh number for the steady instability) and $\mathcal{R}_{o}$ (critical thermal Rayleigh number for the oscillatory instability) versus $P e$ (Peclet number).

\section{Mathematical Model}

Let $\mathrm{L}$ be a horizontal porous layer of depth $d$ through which a homogeneous incompressible electrically conducting fluid moves under the action of an imposed uniform magnetic field $\mathbf{H}$, normal to the layer. Let us assume that $\mathrm{L}$ is uniformly heated from below and salted from below by one chemical $S$. Introducing $O x y z$, an orthogonal frame of reference with fundamental unit vectors $\mathbf{i}, \mathbf{j}, \mathbf{k}$ ( $\mathbf{k}$ pointing vertically upwards), the Darcy model for the motion of the binary fluid mixture, according to the Boussinesq approximation, are (see $[1,10]$ and the references therein): 


$$
\left\{\begin{array}{l}
\frac{v}{k_{p}} \mathbf{v}=-\frac{1}{\rho_{0}} \nabla P^{*}+\frac{\mu_{m}}{4 \pi \rho_{0}} \mathbf{H} \cdot \nabla \mathbf{H}-\left[1-\alpha_{T}\left(T-T_{0}\right)+\alpha_{C}\left(C-C_{0}\right)\right] g \mathbf{k} \\
\frac{\partial \mathbf{H}}{\partial t}+\mathbf{v} \cdot \nabla \mathbf{H}-\mathbf{H} \cdot \nabla \mathbf{v}=\eta \Delta \mathbf{H} \\
\sigma^{*} \frac{\partial T}{\partial t}+\mathbf{v} \cdot \nabla T=k_{T} \Delta T \\
\Phi \frac{\partial C}{\partial t}+\mathbf{v} \cdot \nabla C=k_{C} \Delta C \\
\nabla \cdot \mathbf{v}=0, \nabla \cdot \mathbf{H}=0
\end{array}\right.
$$

with

$$
P^{*}=p+\frac{\mu_{m}|\mathbf{H}|^{2}}{8 \pi}
$$

and

$$
\begin{aligned}
& \mathbf{v}=(U, V, W)=\text { seepage velocity, } T=\text { temperature, } p=\text { pressure, } \\
& \mathbf{H}=\left(H_{1}, H_{2}, H\right)=\text { magnetic field, } C=\text { salt } S \text { concentration, } \mathbf{g}=-g \mathbf{k}=\text { gravity, } \\
& \Phi=\text { porosity, } T_{0}=\text { reference temperature, } C_{0}=\text { reference salt concentration, } \\
& \rho_{0}=\text { reference (constant) density, } \mu_{m}=\text { magnetic permeability, } \eta=\text { resistivity, } \\
& v=\text { kinematic viscosity, } k_{p}=\text { permeability of the medium, } \\
& \alpha_{T}=\text { thermal expansion coefficient, } \alpha_{C}=\text { salt expansion coefficient, } \\
& \sigma^{*}=(\rho c)_{m} /\left(\rho c_{p}\right)_{f}, c=\text { specific heat, } c_{p}=\text { specific heat at a constant pressure, } \\
& k_{T}=\frac{k_{T}^{*}}{\left(\rho c_{p}\right)_{f}}, k_{T}^{*}=\text { effective thermal diffusivity, } \\
& k_{C}=\frac{k_{C}^{*}}{\left(\rho c_{p}\right)_{f}}, k_{C}^{*}=\text { effective salt diffusivity, }
\end{aligned}
$$

where the subscript $m$ and $f$ refer to the medium and fluid respectively. To (1) we append the boundary conditions

$$
\left\{\begin{array}{l}
T(x, y, 0, t)=T_{L}, \quad T(x, y, d, t)=T_{U}, \quad T_{L}>T_{U} \\
C(x, y, 0, t)=C_{L}, \quad C(x, y, d, t)=C_{U}, \quad C_{L}>C_{U} \\
W(x, y, 0, t)=W(x, y, d, t)=q, H(x, y, 0, t)=H(x, y, d, t)=H_{0}
\end{array}\right.
$$

being $q, H_{0}$ constants. The boundary value problem (1) and (2) admits the steady state $m^{*}=$ $\left(p^{*}, \mathbf{v}^{*}, T^{*}, C^{*}, \mathbf{H}^{*}\right)$ given by

$$
\left\{\begin{array}{l}
\mathbf{v}^{*}=q \mathbf{k}, \quad T^{*}(z)=\frac{\left(T_{L}-T_{U}\right) \exp \left[q z / k_{T}\right]+T_{U}-T_{L} \exp \left[d q / k_{T}\right]}{1-\exp \left[d q / k_{T}\right]} \\
C^{*}(z)=\frac{\left(C_{L}-C_{U}\right) \exp \left[q z / k_{C}\right]+C_{U}-C_{L} \exp \left[d q / k_{C}\right]}{1-\exp \left[d q / k_{C}\right]} \\
\mathbf{H}^{*}=H_{0} \mathbf{k}
\end{array}\right.
$$

and $p^{*}(z)$ solution of $(1)_{1}$. Introducing the perturbation fields $\{\pi, \mathbf{u}, \theta, \mathbf{h}, \Gamma\}$ given by

$$
\mathbf{u}=\mathbf{v}-\mathbf{v}^{*}, \mathbf{h}=\mathbf{H}-\mathbf{H}^{*}, \theta=T-T^{*}, \Gamma=C-C^{*}, \pi=p-p^{*},
$$


applying the transformation

$$
\left\{\begin{array}{l}
t=\frac{\sigma^{*} d^{2}}{k_{T}} t^{\prime}, \mathbf{u}=\frac{k_{T}}{d} \mathbf{u}^{\prime}, \mathbf{x}=d \mathbf{x}^{\prime}, \mathbf{h}=\tilde{H} \mathbf{h}^{\prime}, \\
\omega=\frac{\Phi}{\sigma^{*}}, \theta=\tilde{T} \theta^{\prime}, \Gamma=\tilde{\Gamma} \Gamma^{\prime}, \pi=\tilde{P} \pi^{\prime}, \tilde{P}=\frac{\rho_{0} v k_{T}}{k_{p}}, \\
\tilde{H}^{2}=\frac{4 \pi \rho_{0} v k_{T}^{2}}{\eta \mu_{m} k_{p}}, \tilde{T}=T_{L}-T_{U}, \tilde{\Gamma}=C_{L}-C_{U},
\end{array}\right.
$$

it follows that the non-dimensional evolution system for $\{\pi, \mathbf{u}, \theta, \mathbf{h}, \Gamma\}$, omitting the primes, is:

$$
\left\{\begin{array}{l}
\mathbf{u}=-\nabla \pi+R_{T} \theta \mathbf{k}-\frac{R_{C}}{L e} \Gamma \mathbf{k}+\tilde{Q} \frac{\partial \mathbf{h}}{\partial z}+P_{m}^{*} \mathbf{h} \cdot \nabla \mathbf{h}, \\
\tilde{P}_{m} \frac{\partial \mathbf{h}}{\partial t}=\tilde{Q} \frac{\partial \mathbf{u}}{\partial z}-Q^{\prime} \frac{\partial \mathbf{h}}{\partial z}+P_{m}^{*} \mathbf{h} \cdot \nabla \mathbf{u}-P_{m}^{*} \mathbf{u} \cdot \nabla \mathbf{h}+\Delta \mathbf{h} \\
\frac{\partial \theta}{\partial t}+\mathbf{u} \cdot \nabla \theta=-\frac{P e \exp [P e z]}{1-\exp [P e]} w-P e \frac{\partial \theta}{\partial z}+\Delta \theta \\
\omega \frac{\partial \Gamma}{\partial t}+\mathbf{u} \cdot \nabla \Gamma=-\frac{P e L e \exp [P e L e z]}{1-\exp [\text { PeLe] }} w-P e \frac{\partial \Gamma}{\partial z}+\frac{\Delta \Gamma}{L e} \\
\nabla \cdot \mathbf{u}=0, \nabla \cdot \mathbf{h}=0
\end{array}\right.
$$

with

$$
\left\{\begin{array}{l}
L e=\frac{k_{T}}{K_{c}}=\text { Lewis number, } \\
R_{T}=\frac{\alpha_{T} g k_{p} d\left(T_{L}-T_{U}\right)}{v k_{T}}=\text { thermal Rayleigh number, } \\
R_{C}=\frac{\alpha_{C} g k_{p} d\left(C_{L}-C_{U}\right)}{v k_{C}}=\text { salt Rayleigh number, } \\
P_{m}=\frac{v}{\eta}=\text { magnetic Prandtl number, } P_{r}=\frac{v}{k_{T}}=\text { Prandtl number, } \\
P_{m}^{*}=\frac{P_{m}}{P_{r}}=\frac{k_{T}}{\eta}, P_{l}=\sqrt{\frac{k_{p}}{d^{2}}}=\text { porous parameter, } \tilde{P}_{m}=\frac{P_{m}^{*}}{\sigma^{*}}=\frac{k_{T}}{\eta \sigma^{*}} \\
\tilde{Q}=Q P_{l}, Q=\sqrt{\frac{\mu_{m} H_{0}^{2} d^{2}}{4 \pi \rho_{0} v \eta}}=\text { Chandrasekhar number, } \\
Q^{\prime}=\frac{q d}{\eta}, P e=\frac{Q^{\prime}}{P_{m}^{*}}=\frac{q d}{k_{T}}=\text { Péclet number. }
\end{array}\right.
$$

To (6) the following initial-boundary conditions are appended (impermeable and perfect conductors boundaries) $[37,38]$

$$
\left\{\begin{array}{l}
\mathbf{u}(\mathbf{x}, 0)=\mathbf{u}_{0}(\mathbf{x}), \quad \pi(\mathbf{x}, 0)=\pi_{0}(\mathbf{x}), \mathbf{h}(\mathbf{x}, 0)=\mathbf{h}_{0}(\mathbf{x}), \\
\theta(\mathbf{x}, 0)=\theta_{0}(\mathbf{x}), \quad \Gamma(\mathbf{x}, 0)=\Gamma_{0}(\mathbf{x}), \\
w=\theta=h_{1}=h_{2}=\frac{\partial h}{\partial z}=0 \quad \text { on } z=0,1,
\end{array}\right.
$$


being $\mathbf{u}=(u, v, w), \mathbf{h}=\left(h_{1}, h_{2}, h\right)$ and $\nabla \cdot \mathbf{u}_{0}=0, \nabla \cdot \mathbf{h}_{0}=0$. Denoting by

$$
\Omega=\left[0,2 \pi / a_{x}\right] \times\left[0,2 \pi / a_{y}\right] \times[0,1]
$$

the periodicity cell, we assume that the perturbations $\left\{u, v, w, h_{1}, h_{2}, h, \pi, \theta, \Gamma\right\}$ belong to $W^{2,2}(\Omega), \forall t \in$ $\mathbb{R}^{+}$and are periodic in the $x$ and $y$ directions of periods $\frac{2 \pi}{a_{x}}, \frac{2 \pi}{a_{y}}$, respectively.

\section{Instability Analysis Via Normal Modes}

To study the instability of the throughflow solution (3), let us neglect the nonlinear terms in (6) and let us denote by $(\nabla \hat{\pi}, \hat{\mathbf{u}}, \hat{\theta}, \hat{\Gamma})$ the solution of the linearized version of (6) and (7). On taking the third components of the double curl of (6) 1 and of the z-derivative of $(6)_{2}$, one obtains

$$
\left\{\begin{array}{l}
\Delta \hat{w}=\tilde{Q} \frac{\partial}{\partial z} \Delta \hat{h}+R_{T} \Delta_{1} \hat{\theta}-\frac{R_{C}}{L e} \Delta_{1} \hat{\Gamma} \\
\tilde{P}_{m} \frac{\partial}{\partial t}\left(\frac{\partial \hat{h}}{\partial z}\right)=\tilde{Q} \frac{\partial^{2} \hat{w}}{\partial z^{2}}-Q^{\prime} \frac{\partial^{2} \hat{h}}{\partial z^{2}}+\Delta\left(\frac{\partial \hat{h}}{\partial z}\right), \\
\frac{\partial \hat{\theta}}{\partial t}=-\frac{P e \exp [P e z]}{1-\exp [P e]} \hat{w}-P e \frac{\partial \hat{\theta}}{\partial z}+\Delta \hat{\theta} \\
\omega \frac{\partial \hat{\Gamma}}{\partial t}=-\frac{P e L e \exp [P e L e z]}{1-\exp [P e L e]} \hat{w}-P e \frac{\partial \hat{\Gamma}}{\partial z}+\frac{\Delta \hat{\Gamma}}{L e} \\
\nabla \cdot \hat{\mathbf{u}}=0, \nabla \cdot \hat{\mathbf{h}}=0,
\end{array}\right.
$$

being $\Delta_{1}=\frac{\partial^{2}}{\partial x^{2}}+\frac{\partial^{2}}{\partial y^{2}}$. On looking for solutions of normal modes type, in view of periodicity in the $x$ and $y$ directions, one has that

$$
\left(\begin{array}{c}
\hat{w}(x, y, z, t) \\
\hat{h}(x, y, z, t) \\
\hat{\theta}(x, y, z, t) \\
\hat{\Gamma}(x, y, z, t)
\end{array}\right)=\left(\begin{array}{c}
\bar{w}(z) \\
\bar{h}(z) \\
\bar{\theta}(z) \\
\bar{\Gamma}(z)
\end{array}\right) e^{-\sigma t+i\left(a_{x} x+a_{y} y\right)}
$$

being $\sigma \in \mathbb{C}$. Setting

$$
a^{2}=a_{x}^{2}+a_{y}^{2}, \quad D=\frac{d}{d z}
$$

one recovers that $\forall \varphi \in\{\hat{w}, \hat{h}, \hat{\theta}, \hat{\Gamma}\}$

$$
\Delta_{1} \varphi=-a^{2} \varphi, \quad \Delta \varphi=\left(D^{2}-a^{2}\right) \varphi .
$$


Substituting (9) in (8), one obtains

$$
\left\{\begin{array}{l}
\left(D^{2}-a^{2}\right) \bar{w}=\tilde{Q}\left(D^{2}-a^{2}\right) D \bar{h}-R_{T} a^{2} \bar{\theta}+\frac{R_{C}}{L e} a^{2} \bar{\Gamma} \\
-\sigma \tilde{P}_{m} D \bar{h}=\tilde{Q} D^{2} \bar{w}-Q^{\prime} D^{2} \bar{h}+\left(D^{2}-a^{2}\right) D \bar{h} \\
-\sigma \bar{\theta}=-\frac{P e \exp [P e z]}{1-\exp [\text { Pe }]} \bar{w}-P e D \bar{\theta}+\left(D^{2}-a^{2}\right) \bar{\theta} \\
-\sigma \omega \bar{\Gamma}=-\frac{P e L e \exp [\text { PeLez }]}{1-\exp [\text { PeLe }]} \bar{w}-P e D \bar{\Gamma}+\frac{\left(D^{2}-a^{2}\right)}{L e} \bar{\Gamma}
\end{array}\right.
$$

under the boundary conditions

$$
\bar{w}=\bar{\theta}=\bar{\Gamma}=D \bar{h}=0, \quad \text { on } z=0,1 .
$$

To determine an approximate solution of (12) and (13), we employ an order-1 Galerkin weighted residuals method [35]. To this end, we choose as trial functions satisfying the boundary conditions (13)

$$
\begin{array}{ll}
\bar{w}=C_{1} \sin \pi z, & \bar{h}=C_{2} \cos \pi z, \\
\bar{\theta}=C_{3} \sin \pi z, & \bar{\Gamma}=C_{4} \sin \pi z,
\end{array}
$$

where $C_{i}$ are constants $i \in\{1,2,3,4\}$. Substituting (14) in (12), we obtain three residuals. Making these residuals orthogonal to the trial functions over the range $0 \leq z \leq 1$, one obtains the following system of four linear algebraic equations in the four unknown $C_{1}, C_{2}, C_{3}, C_{4}$

$$
\left\{\begin{array}{l}
\left(a^{2}+\pi^{2}\right) C_{1}+\pi \tilde{Q}\left(a^{2}+\pi^{2}\right) C_{2}-R_{T} a^{2} C_{3}+\frac{R_{C}}{L e} a^{2} C_{4}=0, \\
\pi \tilde{Q} C_{1}-\left(a^{2}+\pi^{2}-\sigma \tilde{P}_{m}\right) C_{2}=0, \\
\frac{4 \pi^{2}}{4 \pi^{2}+P e^{2}} C_{1}-\left(a^{2}+\pi^{2}-\sigma\right) C_{3}=0, \\
\frac{4 \pi^{2}}{4 \pi^{2}+L e^{2} P e^{2}} C_{1}-\left(\frac{a^{2}+\pi^{2}}{L e}-\sigma \omega\right) C_{4}=0 .
\end{array}\right.
$$

Requiring the vanishing of the determinant of the coefficient matrix, one obtains the approximate instability threshold

$$
\mathcal{R}_{c}\left(a^{2}, \sigma\right):=\frac{4 \pi^{2}+P e^{2}}{4 \pi^{2}+L e^{2} P e^{2}} \frac{\xi-\sigma}{\xi-L e \sigma \omega} R_{C}+\frac{\left(4 \pi^{2}+P e^{2}\right) \xi(\xi-\sigma)}{4 \pi^{2} a^{2}}+\frac{4 \pi^{2}+P e^{2}}{4} \frac{(\xi-\sigma) \xi \tilde{Q}^{2}}{a^{2}\left(\xi-\sigma \tilde{P}_{m}\right)}
$$

with $\xi=a^{2}+\pi^{2}$. The meaning of (16) is the following. Once fixed $a^{2} \in \mathbb{R}^{+}$, for $\mathcal{R}_{T}=\mathcal{R}_{c}$ the throughflow (3) loses its stability and a secondary motion, steady or unsteady, arises. We remark that, by numerical computation, this is a good approximation for $P e \leq 1$.

The critical Rayleigh thermal number at which a secondary steady motion arises when (3) becomes unstable (say $\mathcal{R}_{s}$ ), is obtained on substituting $\sigma=0$ in (16) and looking for the minimum with respect to $a^{2} \in \mathbb{R}^{+}$, i.e.,

$$
\mathcal{R}_{s}=\min _{a^{2} \in \mathbb{R}^{+}} f\left(a^{2}\right),
$$

with

$$
f\left(a^{2}\right):=\frac{4 \pi^{2}+P e^{2}}{4 \pi^{2}+L e^{2} P e^{2}} R_{C}+\frac{\left(4 \pi^{2}+P e^{2}\right) \xi}{4 a^{2}} \tilde{Q}^{2}+\frac{4 \pi^{2}+P e^{2}}{4 \pi^{2}} \frac{\xi^{2}}{a^{2}}
$$


Simple calculation shows that

$$
\min _{a^{2} \in \mathbb{R}^{+}} f\left(a^{2}\right)=f\left(a_{c}^{2}\right)
$$

with

$$
a_{c}^{2}=\pi^{2} \sqrt{1+\tilde{Q}^{2}}
$$

and hence $\mathcal{R}_{s}$ is given by

$$
\mathcal{R}_{s}=\frac{4 \pi^{2}+P e^{2}}{4 \pi^{2}+L e^{2} P e^{2}} R_{C}+\frac{4 \pi^{2}+P e^{2}}{4} \frac{1+\sqrt{1+\tilde{Q}^{2}}}{\sqrt{1+\tilde{Q}^{2}}} \tilde{Q}^{2}+\frac{4 \pi^{2}+P e^{2}}{4} \frac{\left[1+\sqrt{1+\tilde{Q}^{2}}\right]^{2}}{\sqrt{1+\tilde{Q}^{2}}} .
$$

The critical Rayleigh number at which a secondary oscillatory motion arises (say $\mathcal{R}_{0}$ ) is obtained on substituting $\sigma=i \sigma_{1}, \sigma_{1} \neq 0$ in (16) and looking for the minimum with respect to $a^{2} \in \mathbb{R}^{+}$. Setting the imaginary part equal to zero it follows that

$$
\frac{4 \pi^{2}+P e^{2}}{4 \pi^{2}+L e^{2} P e^{2}} \frac{1-\omega L e}{\xi^{2}+\omega^{2} L e^{2} \sigma_{1}^{2}} R_{C}+\frac{4 \pi^{2}+P e^{2}}{4 \pi^{2} a^{2}}+\frac{\left(4 \pi^{2}+P e^{2}\right) \xi \tilde{Q}^{2}}{4 a^{2}} \frac{1-\tilde{P}_{m}}{\xi^{2}+\tilde{P}_{m}^{2} \sigma_{1}^{2}}=0
$$

From (22), it easily follows that, when

$$
\omega L e \leq 1, \quad \tilde{P}_{m} \leq 1,
$$

(22) does not admit any solutions, i.e., instability can occur only via a steady state. When (23) is not satisfied, then, denoting by $\sigma_{*}$ the positive root of (22), one has that

$$
\mathcal{R}_{o}=\min _{a^{2} \in \mathbb{R}^{+}} g\left(a^{2}\right),
$$

with

$$
g\left(a^{2}\right):=\frac{4 \pi^{2}+P e^{2}}{4 \pi^{2}+L e^{2} P e^{2}} \frac{\xi^{2}+\omega L e \sigma_{*}^{2}}{\xi^{2}+\omega^{2} L e^{2} \sigma_{*}^{2}} R_{c}+\frac{\left(4 \pi^{2}+P e^{2}\right) \xi \tilde{Q}^{2}}{4 a^{2}} \frac{\xi^{2}+\tilde{P}_{m} \sigma_{*}^{2}}{\xi^{2}+\tilde{P}_{m}^{2} \sigma_{*}^{2}}+\frac{\left(4 \pi^{2}+P e^{2}\right) \xi^{2}}{4 \pi^{2} a^{2}}
$$

From (18) and (25), it follows that in the case

$$
\omega L e>1, \quad \tilde{P}_{m}>1,
$$

one has

$$
g\left(a^{2}\right)<f\left(a^{2}\right), \forall a^{2} \in \mathbb{R}^{+}
$$

and hence $\mathcal{R}_{o}<\mathcal{R}_{s}$. This means that, when (26) holds, the secondary motion arising when (3) becomes unstable, is oscillatory.

We remark that condition (23) continues to be a sufficient condition for the onset of steady instability when the fluid is, initially, at the rest state [10]. Let us assume that (23) holds and let us compare $\mathcal{R}_{s}$ in (21) with the critical Rayleigh thermal numbers in $[10,22]$ found, respectively, in the absence of throughflow and in the absence of the magnetic field. When $\mathrm{Pe} \rightarrow 0$ (negligible throughflow), one has that

$$
\lim _{P e \rightarrow 0} \mathcal{R}_{s}=R_{C}+\pi^{2}\left[1+\sqrt{1+\tilde{Q}^{2}}\right]^{2}
$$

that is the critical Rayleigh thermal number for the onset of steady convection found in [10]. Analogously, when $\tilde{Q}^{2} \rightarrow 0$ (negligible magnetic field), one has that

$$
\lim _{\tilde{Q}^{2} \rightarrow 0} \mathcal{R}_{s}=\frac{4 \pi^{2}+P e^{2}}{4 \pi^{2}+L e^{2} P e^{2}} R_{c}+4 \pi^{2}+P e^{2},
$$


that is the critical Rayleigh thermal number for the onset of steady instability of a vertical constant throughflow in a binary mixture saturating a horizontal porous layer, found in [22].

In the case (23), in order to look for the influence of the throughflow on the onset of instability, let us remark that

$$
\frac{\partial \mathcal{R}_{s}}{\partial P e}=R_{C} \frac{4 \pi^{2}\left(1-L e^{2}\right)}{\left(4 \pi^{2}+L e^{2} P e^{2}\right)^{2}}+\frac{\left[1+\sqrt{1+\widetilde{Q}^{2}}\right]^{2}}{4},
$$

and hence if $\left(1-L e^{2}\right) \geq 0$, then the throughflow has a stabilizing effect on the onset of steady instability. Analogously, in order to look for the influence of the magnetic field on the onset of instability, one has that

$$
\frac{\partial \mathcal{R}_{s}}{\partial \tilde{Q}^{2}}=\frac{4 \pi^{2}+P e^{2}}{4}\left(1+\frac{1}{\sqrt{1+\tilde{Q}^{2}}}\right)>0,
$$

i.e., the magnetic field has a stabilizing effects, as one is expected.

\section{Conclusions}

The instability of a vertical constant throughflow in a horizontal porous layer embedded in an external constant magnetic field and filled by an electrically conducting double fluid mixture, is performed. The order-1 Galerkin weighted residuals method, has been employed to determine the critical Rayleigh numbers for the onset of steady and oscillatory instability. This method provides a good approximation for $P e \leq 1$. Sufficient conditions for the occurrence of steady and oscillatory instability have been found. In the following Table 1, some values of the constant parameters appearing in (6) have been fixed, in order to show some examples in which steady or oscillatory instability sets in.

Table 1. Onset of steady or oscillatory instability for some particular values of $\tilde{P}_{m}, L e, \omega, \tilde{Q}^{2}, P e, R_{C}$.

\begin{tabular}{ccccccccc}
\hline$\tilde{\boldsymbol{P}}_{\boldsymbol{m}}$ & $\boldsymbol{L} \boldsymbol{e}$ & $\boldsymbol{\omega}$ & $\tilde{Q}^{\mathbf{2}}$ & $\boldsymbol{P e}$ & $\boldsymbol{R}_{\boldsymbol{C}}$ & $\boldsymbol{R}_{\boldsymbol{S}}$ & $\boldsymbol{R}_{\boldsymbol{O}}$ & Instability \\
\hline 0.5 & 1 & 0.2 & 10 & 0.5 & 50 & 235.067 & - & Steady \\
1 & 1.5 & 0.2 & 0.3 & 0.2 & 50 & 95.1889 & $17,107.7$ & Steady \\
2.5 & 2 & 0.5 & 0.001 & 0.4 & 50 & 89.06 & 47.2763 & Oscillatory \\
\hline
\end{tabular}

Furthermore, the stabilizing effect of the vertical constant throughflow for the onset of steady convection, has been examined. In Figure 1, the stabilizing effect of the vertical constant throughflow on the onset of instability, is showed.
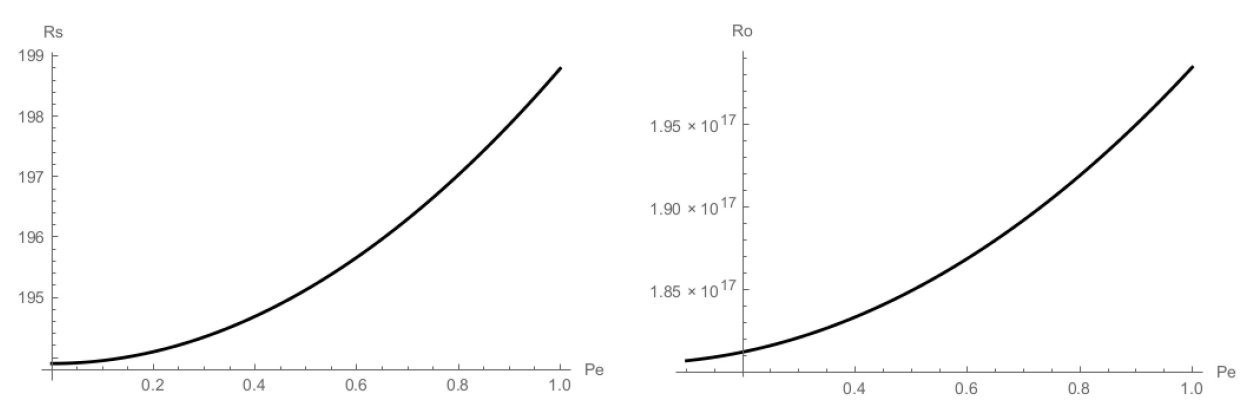

Figure 1. Stabilizing effect of the vertical constant throughflow when $\left\{\tilde{P}_{m}=1, L e=0.3, \omega=0.5, \tilde{Q}^{2}=\right.$ 10, $\left.R_{C}=10\right\}$. Left: behaviour of $R_{S}$ versus $P e$; Right: behaviour of $R_{O}$ versus $P e$.

Author Contributions: The authors conceived the mathematical model, proved the mathematical results and wrote the paper together. The authors gave their final approval for publication.

Funding: This research received no external funding.

Acknowledgments: The paper has been performed under the ausipces of GNFM of INdAM. One of Author (R. De Luca) also thanks Progetto Giovani GNFM 2019 “Modellazione ed analisi di sistemi microbici complessi: 
applicazione ai biofilm". The Authors should like to thank anonymous referees for suggestions which have led to improvements in the manuscript.

Conflicts of Interest: The authors declare no conflict of interest.

\section{References}

1. Nield, D.A.; Bejan, A. Convection in Porous Media; Springer: Berlin/Heidelberg, Germany; New York, NY, USA, 2017.

2. Barletta, A.; Celli, M. Convective to Absolute Instability Transition in a Horizontal Porous Channel with Open Upper Boundary. Fluids 2017, 2, 1-22.

3. Bidin, B.; Rees, D.A.S. The Onset of Convection in an Unsteady Thermal Boundary Layer in a Porous Medium. Fluids 2016, 1, 41. [CrossRef]

4. Braga, N.R.; Brandao, P.V.; Alves, L.S.D.B.; Barletta, A. Convective instability induced by internal and external heating in a fluid saturated porous medium. Int. J. Heat Mass Transf. 2017, 108, 2393-2400. [CrossRef]

5. Chamkha, A.J. Double-diffusive convection in a porous enclosure with cooperating temperature and concentration gradients and heat generation or absorption effects. Numer. Heat Transf. Part A Appl. 2002, 41, 65-87. [CrossRef]

6. Rees, D.A.S.; Barletta, A. Onset of convection in a porous layer with continuous periodic horizontal stratification, Part II: Three-dimensional convection. Eur. J. Mech. B Fluids 2014, 47, 57-67. [CrossRef]

7. Rees, D.A.S.; Nield, D. The Effect of an Embedded Solid Block on the Onset of Convection in a Porous Cavity. Int. J. Numer. Methods Heat Fluid Flow 2016, 26, 950-976. [CrossRef]

8. Storesletten, L.; Rees, D.A.S. Onset of Convection in an Inclined Anisotropic Porous Layer with Internal Heat Generation. Fluids 2019, 4, 75. [CrossRef]

9. Capone, F.; De Luca, R. Porous MHD convection: Effect of Vadasz inertia term. Transp. Porous Med. 2017, 118, 519-536. [CrossRef]

10. Capone, F.; De Luca, R. Double diffusive convection in porous media under the action of a magnetic field. Ricerche Mat. 2018. [CrossRef]

11. Chamkha, A.J. On laminar hydromagnetic mixed convection flow in a vertical channel with symmetric and asymmetric wall heating conditions. Int. J. Heat Mass Transf. 2002, 5 2509-2525. [CrossRef]

12. Chamkha, A.J. Hydromagnetic natural convection from an isothermal inclined surface adjacent to a thermally stratified porous medium. Int. J. Eng. Sci. 1997, 35, 975-986. [CrossRef]

13. Capone, F.; Rionero, S. Porous MHD convection: Stabilizing effect of magnetic field and bifurcation analysis. Ricerche Mat. 2016, 65, 163-186. [CrossRef]

14. Capone, F.; Rionero, S. Brinkmann viscosity action in porous MHD convection. Int. J. Non-Linear Mech. 2016, 85, 109-117. [CrossRef]

15. Rionero, S. Dynamic of thermo-MHD flows via a new approach. Atti Accad. Naz. Lincei Cl. Sci. Fis. Mat. Natur. Rend. Lincei 2017, 28, 21-47. [CrossRef]

16. Barletta, A.; Rossi di Schio E.; Storesletten, L. Convective roll instabilities of vertical throughflow with viscous dissipation in a horizontal porous layer. Transp. Porous Media 2010, 3, 461-477. [CrossRef]

17. Barletta, A.; Storesletten, L. Linear instability of the vertical throughflow in a horizontal porous layer saturated by a power-law fluid. Int. J. Heat Mass Transf. 2016, 99, 293-302. [CrossRef]

18. Bharti, S.P.K.; Sharma, R.C. On Bénard convection in a porous medium in the presence of throughflow and rotation in hydromagnetics. Arch. Mech. (Archiwum Mechaniki Stosowanej) 2003, 55, 257-274.

19. Capone, F.; De Cataldis, V.; De Luca R.; Torcicollo, I. On the stability of vertical constant throughflows for binary mixtures in porous layers. Int. J. Non-Linear Mech. 2014, 59, 1-8. [CrossRef]

20. Capone, F.; De Luca, R. On the stability-instability of vertical throughflows in double diffusive mixtures saturating rotating porous layers with large pores. Ricerche di Matematica 2014, 63, 119-148. [CrossRef]

21. Capone, F.; De Luca, R.; Torcicollo, I. Longtime behavior of vertical throughflows for binary mixtures in porous layers. Int. J. Non-Linear Mech. 2013, 52, 1-7. [CrossRef]

22. Capone, F.; De Luca, R.; Torcicollo, I. Instability of Vertical Constant Through Flows in Binary Mixtures in Porous Media with Large Pores. Math. Probl. Eng. 2019. [CrossRef]

23. Capone, F.; De Luca, R. Coincidence between linear and global nonlinear stability of non-constant throughflows via the Rionero "Auxiliary System Method". Meccanica 2014, 49, 2025-2036. [CrossRef] 
24. Chen, F. Throughflow effects on convective instability in superposed fluid and porous layers. J. Fluid Mech. 1991, 231, 113-133. [CrossRef]

25. Hill, A.A.; Rionero S.; Straughan, B. Global stability for penetrative convection with throughflow in a porous material. IMA J. Appl. Math. 2007, 72, 635-643. [CrossRef]

26. Kiran, P. Throughflow and non-uniform heating effects on double diffusive oscillatory convection in a porous medium. Ain Shams Eng. J. 2016, 7, 453-462. [CrossRef]

27. Murty, Y.N. Effect of throughflow and magnetic field on Bénard convection in micropolar fluids. Acta Mech. 2001, 150, 11-21. [CrossRef]

28. Shivakumara, I.S.; Khalili, A. On the stability of double diffusive convection in a porous layer with throughflow. Acta Mech. 2001, 152, 165-175. [CrossRef]

29. Sutton, F.M. Onset of convection in a porous channel with net through flow. Phys. Fluids 1970, 13, 1931. [CrossRef]

30. Nield, D.A. Throughflow effects on the Rayleigh-Bénard convective instability problem. J. Fluid Mech. 1987, 185, 353-360. [CrossRef]

31. Nield, D.A.; Kuznetsov, A.V. The Effect of Vertical Throughflow on the Onset of Convection in a Porous Medium in a Rectangular box. Transp. Porous Media 2011, 90, 993-1000. [CrossRef]

32. Shivakumara, I.S.; Suma, S.P. Rayleigh-Bénard convection with throughflow: A comparative study between the single-term and higher order Gelerkin methods. Appl. Mech. Eng. 2000, 5, 507-520.

33. Zhao, C.; Hobbs, B.E.; Muhlhaus, H.B. Theoretical and numerical analysis of convective instability in porous media with upward throughflow. Int. J. Numer. Anal. Methods Geomech. 1999, 23, 629-646. [CrossRef]

34. Nield, D.A.; Bejan, A. Convection in Porous Media, 5th ed.; Springer: Berlin, Germany, 2017.

35. Nield, D.A.; Kuznetsov, A.V. Onset of convection in a porous medium with strong vertical throughflow. Transp. Porous Media 2011, 90, 883-888. [CrossRef]

36. Qiao, Z.; Kaloni, P.N. Convection in a Porous Medium Induced by an Inclined Temperature Gradient With Mass Flow. J. Heat Transf. 1997, 119, 366-370. [CrossRef]

37. Chandrasekhar, S. Hydrodynamic and Hydromagnetic Stability; Dover: Mineola, NY, USA, 1981.

38. Mulone, G.; Rionero, S. Necessary and sufficient conditions for nonlinear stability in the Magnetic Bénard Problem. Arch. Rational Mech. Anal. 2003, 166, 197-218. [CrossRef] 